\title{
Post-Chlorination Process Control based on Flow Prediction by Time Series Neural Network in Water Treatment Plant
}

HoHyun Lee ${ }^{1}$, GangWook Shin ${ }^{2}$, SungTaek Hong ${ }^{2}$, JongWoong $\mathrm{Choi}^{2}$, and MyungGeun Chun ${ }^{1}$

${ }^{1}$ School of Electrical Engineering and Computer Science, Chungbuk National University, Cheongju, Korea ${ }^{2}$ K-water Research Institute, Korea Water Resources Corporation, Daejeon, Korea

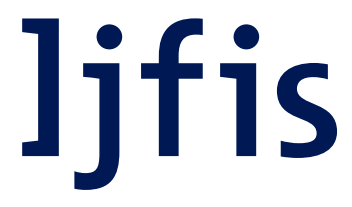

Received: Aug. 16, 2016

Revised : Sep. 12, 2016

Accepted: Sep. 13, 2016

Correspondence to: MyungGeun Chun (mgchun@cbnu.ac.kr)

(The Korean Institute of Intelligent Systems

(c)This is an Open Access article distributed under the terms of the Creative Commons Attribution Non-Commercial License (http://creativecommons.org/licenses/ by-nc/3.0// which permits unrestricted noncommercial use, distribution, and reproduction in any medium, provided the original work is properly cited.

\begin{abstract}
It is very important to maintain a constant chlorine concentration in the post chlorination process, which is the final step in the water treatment process (hereafter WTP) before servicing water to citizens. Even though a flow meter between the filtration basin and clear well must be installed for the post chlorination process, it is not easy to install owing to poor installation conditions. In such a case, a raw water flow meter has been used as an alternative and has led to dosage errors due to detention time. Therefore, the inlet flow to the clear well is estimated by a time series neural network for the plant without a measurement value, a new residual chlorine meter is installed in the inlet of the clear well to decrease the control period, and the proposed modeling and controller to analyze the chlorine concentration change in the well is a neuro fuzzy algorithm and cascade method. The proposed algorithm led to post chlorination and chlorination improvements of 1.75 times and 1.96 times respectively when it was applied to an operating WTP. As a result, a hygienically safer drinking water is supplied with preemptive response for the time delay and inherent characteristics of the disinfection process.
\end{abstract}

Keywords: Time series, Neuro-fuzzy, Water treatment plant, Residual chlorine

\section{Introduction}

Tap water shall maintain adequate disinfection capability values in order to ensure the safety of drinking water from various water-borne diseases caused by bacteria, viruses and protists. According to the water treatment standards enacted in 2002 by the Ministry of Environment, inactivation targets of viruses and giardia are more than 4-log and 3-log, each of which corresponds to the deactivation rate of $99.99 \%, 99.9 \%$, respectively [1]. Target chlorine concentration rates could be different depending on raw water quality, pre-processing and storage tank structure to ensure adequate disinfection capability. But around $1.0 \mathrm{mg} / \mathrm{L}$ residual chlorine and a residence time 2 to 5 hours in the storage tank is typically maintained [2]. Even though maintenance of adequate residual chlorine concentration is necessarily required for secondary pollution control and microbial regrowth inhibition in the water supply process, domestic water supply systems have a national average leakage rate of $15 \%$, which could possibly introduce a variety of contaminants into the pipes as a result of pressure decreases [3]. To prevent penetration, disinfection equipment must be installed regardless of its processing 
method and size. An inexpensive chlorine for the disinfection is most typically used to satisfy the conditions of the residual chlorine in tap water [4].

General disinfecting control employs a method of determining the dosage quantity by multiplying the influent flow with the dosage rate value set in the SCADA (Supervisory Control and Data Acquisition) system. The chlorine injection rate is manually changed by an experienced worker or automatically changed by the feedback control in order to maintain the residual chlorine amount in case of raw water quality variations $[5$, 6]. While a residual chlorine concentration prediction model was developed for the pre-chlorination process [7] and the distribution network [8], a model for the post-chlorination process has not been presented.

In the post chlorination, there are three important factors to keep the chlorine rate constant, which are flow rate, residual chlorine and dosage rate. Among them, some plants don't have the flow meter at the point of post chlorination and replace it with raw water flow. In that case, the dosage quantity is inevitably miscalculated according to wrong flow information by residence time in former chambers and backwashing. Thus, this paper proposes the inlet flow to clear well is estimated by time series neural network for the plant without a measurement value. In addition, the statistical analysis are considered for the estimation of the residual chlorine change and the exact dosage rate based on historical database in WTP.

\section{Algorithm for Post-Chlorination}

In this paper, we propose the method of filtration flow prediction and post-chlorination modeling based on historical data in order to maintain a constant chlorine rate by determining the optimal dosage rate. Also, cascade control is added to overcome the effects of delay time and environment by changing the input target value according to the output residual chlorine rate.

\subsection{Challenges in Post-Chlorination Process}

Figure 1 shows the general water treatment process in which river water is sent to a water treatment plant using intake pumps and purified by sequential processes. Chemicals such as chlorine and alum are added to the influent water and it goes through the process of coagulation, sedimentation, filtration and disinfection for post-chlorination. Once chlorine disinfection is committed after the filtration process, water arrives at the storage tank to be mixed properly. The disinfected effluent water is supplied to the general public through pipelines.

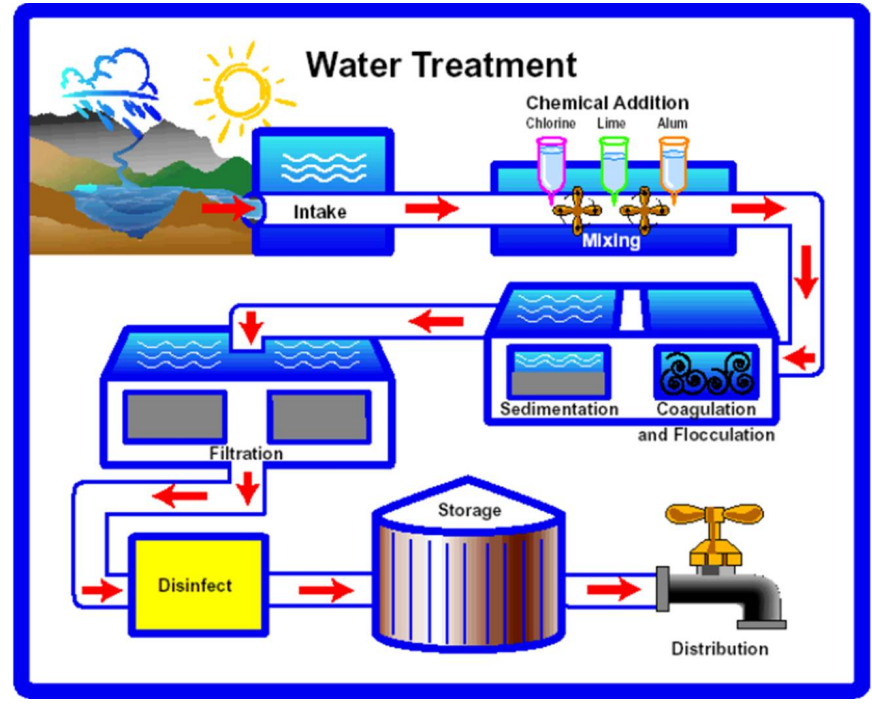

Figure 1. General water treatment process.

\subsubsection{Difficulty of plant modeling}

In order to estimate the inflow of a storage tank, hydraulic modeling is required for coagulation, sedimentation, and filtration processes. Besides, there is no formula to determine the chlorine injection rate by understanding the variation of residual chlorine concentration in accordance with various factors, which are very difficult to model with the continuous changes in water quality and weather $[9,10]$.

\subsubsection{Uninstallation of flow meters}

It is very important to know the flow at the point where the chlorine is injected to determine the exact quantity of the chemical. Unfortunately, many water purification plants do not have a meter owing to short straight pipelines, insufficient water in the pipe, too many bubbles and so on. Therefore, raw water flow is considered instead, which is installed as far away as 1 $\mathrm{km}$ or so and leads to the disturbance of the chlorine dosage by difference with the true flow value in the injection point. Eq. (1) is a basic formula for the chlorine injection amount. The chlorine dosage is calculated by multiplying the chlorine injection rate and the flow, which shows that the flow is a very important factor [11].

$$
V_{w}=Q \times R \times 10^{-3} .
$$

$V_{w}:$ Chlorine Injection Quantity $(\mathrm{kg} / \mathrm{h})$

$Q:$ Hourly Flow $\left(\mathrm{m}^{3} / \mathrm{h}\right)$

$R$ : Chlorine Dosing Rate(mg/L) 


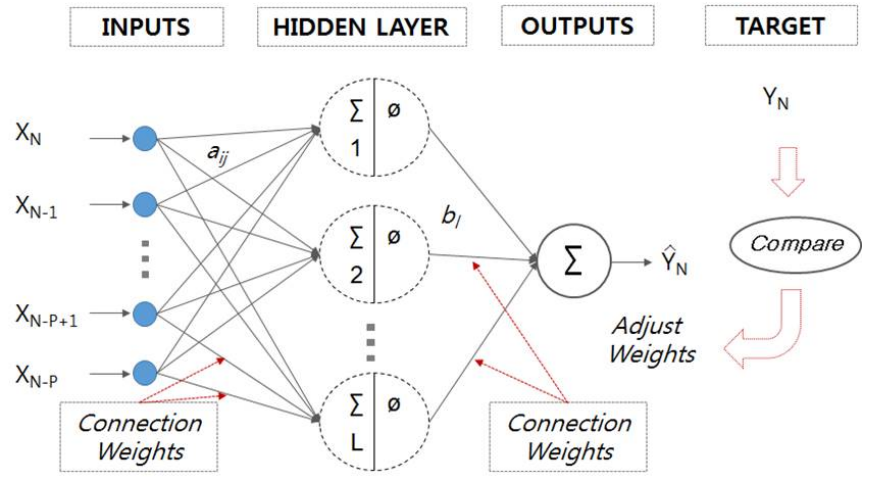

Figure 2. Structure of a time-series neural network.

\subsubsection{Detention time in the storage tank}

It is difficult for feedback control to keep the output residual chlorine constant, because the reaction time may take 2-6 hours by water usage. Thus, a new chlorine meter is installed in the inlet of the storage tank to provide feedback control. However, chlorine evaporation can also be different depending on water demand [12]. Data mapping should be preceded in accordance with the residence time for data analysis.

\subsection{Reviewed Algorithms}

\subsubsection{Time series neural network}

A time series is a series of data points listed in time order. Time series analysis comprises methods for analyzing time series data in order to extract meaningful statistics and other characteristics. Time series neural network is a model to estimate time series date by neural network. The neural networks are known to be particularly effective for discontinuous time series data.

Flow prediction was examined by the time series neural. Neural networks to mimic the brain's information processing capabilities have been studied since Rosenblatt applied the first single-layer perceptron pattern classification problems in 1950. But Minsky pointed out that a single-layer perceptron cannot implement the XOR logic, which caused the recession of the neural network learning algorithms for a while. Then, it has been revitalized again with multi-layer perceptron, analog large scale integrated circuits, and parallel processing techniques [13-15].

Figure 2 is a structural diagram of the time series neural net with a delay of $\mathrm{P}$ minutes to predict the current filtration flow rate $\left(\hat{Y}_{N}\right)$ considering raw water flows $\left(X_{N} \sim X_{N-P}\right)$.

The procedure consists of five steps to learn weight in each step, and the step-by-step procedure is as follows:
Step 1: Specify connection weights between the neurons $\mathrm{N}$ by using the M pattern, as shown in Eq. (2).

$$
W_{i, j}=\left\{\begin{array}{l}
\sum_{s=0}^{M-1} X_{i}^{s} X_{j}^{s}, \quad i \neq j \\
0 \quad, \quad i=j
\end{array} \quad(0 \leq i, j \leq N-1) .\right.
$$

$W_{i, j}$ : Connection weight between neuron $i$ to $j$

$X_{i}^{s}: i$-th value of learning pattern pertaining in class $(+1$ or $-1)$

Step 2: Present an input pattern of the neural network image, as shown in Eq. (3).

$$
\mu_{i}(0)=X_{i}, \quad(0 \leq i, j \leq N-1)
$$

Step 3: Pass the transfer function by adding the value multiplied by output values and weights of the neurons, as shown in Eq. (4).

$$
\mu_{i}(t+1)=f_{h}\left(\sum_{i=0}^{N-1} \mu_{i}(t) * W_{i, j}\right), \quad(0 \leq i, j \leq N-1),
$$

where $f_{h}(a)=\left\{\begin{aligned} 1, & \text { if } a \geq 0, \\ -1, & \text { if } a<0 .\end{aligned}\right.$

Step 4 : Repeat Step 3 before convergence (no change in output).

Step 5: Branch to Step 2.

\subsubsection{Neuro fuzzy algorithm}

An adaptive neuro-fuzzy inference system or adaptive networkbased fuzzy inference system (ANFIS) is a kind of artificial neural network that is based on Takagi-Sugeno fuzzy inference system. The technique was developed in the early 1990s. Since it integrates both neural networks and fuzzy logic principles, it has potential to capture the benefits of both in a single framework. Its inference system corresponds to a set of fuzzy IF-THEN rules that have learning capability to approximate nonlinear functions.

ANFIS was adopted as a learning algorithm for the controller of optimal chlorine dosage rate and modeling of chlorine rate changes in the clear well, which was also used in the estimation of density level for effluent treatment process [16]. It operates well using a small number of rules compared to other neural networks. ANFIS is known as one of the methods to organize the fuzzy inference system with the given input-output data pairs. The parameters of consequent parts can be optimized 


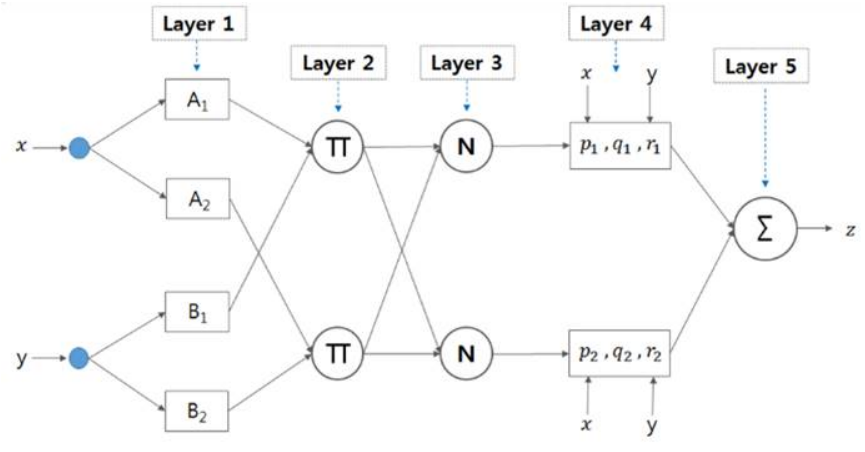

Figure 3. ANFIS structure.

using the least square method while the premise parameters using the steepest descent method. It is assumed that the fuzzy inference system has two inputs, $\mathrm{x}, \mathrm{y}$ and one output $\mathrm{z}$.

Rule 1: If $\mathrm{x}$ is $A_{1}$ and $\mathrm{y}$ is $B_{1}$, then $f_{1}=p_{1} x+q_{1} y+r_{1}$

Rule 2: If $\mathrm{x}$ is $A_{2}$ and $\mathrm{y}$ is $B_{2}$, then $f_{2}=p_{2} x+q_{2} y+r_{2}$

A detailed description of an ANFIS structure is summarized in Figure 3. A square node (adaptive node) has parameters, while a circle node (fixed node) has none. The node functions in the same layer are of the same family function as described in Figure 3:

Layer 1: Every node $\mathrm{i}$ in the first layer is an adaptive node with node function as shown in Eq. (5).

$$
O_{i}^{1}=u_{A_{i}}(x), O_{i}^{1}=u_{B_{i}}(y),
$$

where $x$ is the input to node $\mathrm{i}$ and $A_{i}$ is the linguistic label (small, large, etc.). In other words, $O_{i}^{1}$ is the membership function of $A_{1}$ and it specifies the degree to which the given $z$ satisfies the quantifier $A_{i}$. A bell-shaped function was used as shown in Eq. (6).

$$
u_{D}(x)=\frac{1}{1+\left|\frac{x-c_{i}}{a_{i}}\right|}{ }^{2 b_{i}} .
$$

Here $\left\{a_{i}, b_{i}, c_{i}\right\}$ is the parameter set. The parameters in this layer are referred to as premise parameters.

Layer 2: Every node in this layer is a fixed node labeled $\Pi$, which multiplies the incoming signals and sends the product as the output, as shown in Eq. (7). For instance,

$$
O_{i}^{2}=w_{i}=u_{A_{i}}(x) \times u_{B_{i}}(y), \quad i=1,2 .
$$

Each node output represents the firing strength of a rule.

Layer 3: Every node in this layer is a fixed node labeled $\mathrm{N}$. The i-th node calculates the ratio of the firing strength of the $i$-th rule to the sum of the firing strengths of all the rules, as shown in Eq. (8)

$$
O_{i}^{3}=\bar{w}_{i}=\frac{w_{i}}{w_{1}+w_{2}}, \quad i=1,2 .
$$

For convenience, the outputs of this layer are referred to as normalized firing strengths.

Layer 4: Every node $\mathrm{i}$ in this layer is an adaptive node with the node function shown in Eq. (9).

$$
O_{i}^{4}=\bar{w}_{i} f_{i}=\bar{w}_{i}\left(p_{i} x+q_{i} y+r_{i}\right), \quad i=1,2,
$$

where, $\bar{w}_{i}$ are normalized firing strengths obtained in Layer 3 and $p_{i}, q_{i}$, and $r_{i}$ are the linear parameters of Tagaki-Sugeno fuzzy modeling. They are referred to as the consequent parameters.

Layer 5: The single node in this layer computes the overall output as the summation of all the incoming signals, as shown in Eq. 10,.

$$
O_{i}^{5}=\sum \bar{w}_{i} f_{i}=\frac{\sum w_{i} f_{i}}{\sum w_{i}} .
$$

The premise and consequent parameters can be chosen to minimize the following sum of the squared error, as shown in Eq. (11).

$$
E=\sum_{m=1}^{N}\left(T_{m}-O_{m}\right)^{2} .
$$

Here, $T_{m}$ is the desired output of the m-th data, $O_{m}$ is the output of the fuzzy model using the $\mathrm{m}$-th data, and $\mathrm{N}$ is the total number of training data sets. The steepest descent method, as in a neural network, can be applied to determine the premise parameters, and the least square estimate can be applied to optimize the consequent parameters [17].

\section{Development and Implementation of an In- telligent Controller}

\subsection{New Residual Chlorine Meter and Cascade Control}

In terms of the conventional chlorination process, the chlorine dosage rate depends on an operator's experience to obtain a desired target residual chlorine rate. Post-chlorination process needs a long reaction time in the clear well, which prevents from keeping the output chlorine concentration constant. Therefore, a new chlorine meter is installed in the inlet of the well as shown in Figure 4.

Then, it is modeled using neuro fuzzy algorithm to know the relationship between the dependent variable (input chlorine 


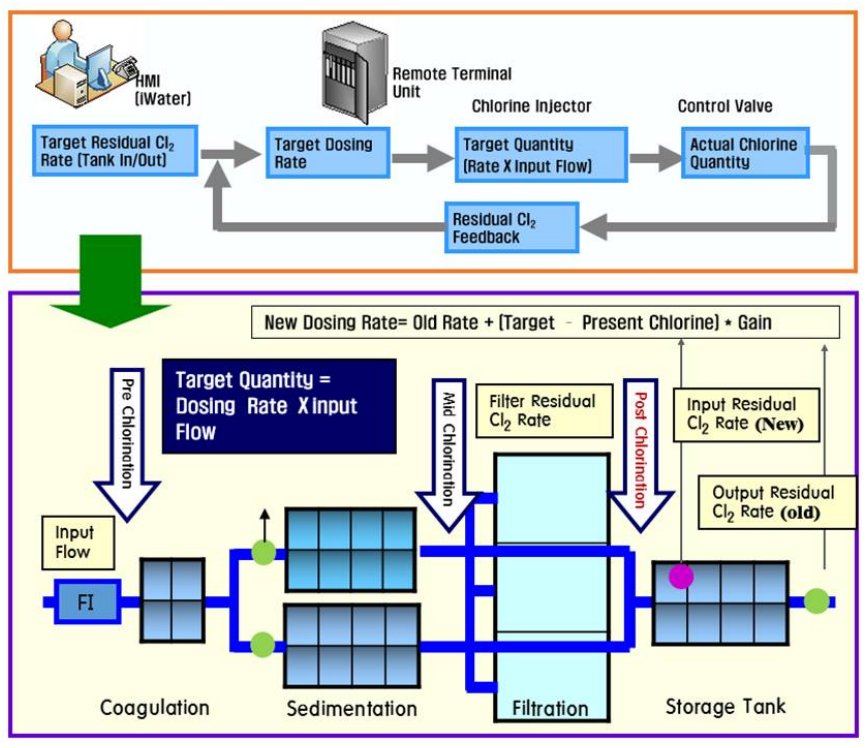

Figure 4. Configuration of post-chlorination process.

rate) and the independent variables (filter output chlorine rate, filter outflow, and dosage rate). In addition, cascade control is added to correct the target input chlorine rate by checking the target output chlorine rate in the storage tank.

\subsection{Implementation of a Hybrid Neuro-Fuzzy Controller}

A cascade control consists of two feedback control loops, in which the output of the first feedback loop is added to the set value of the secondary loop. It is very appropriate for the chlorine process to have a long time delay. As the filtration flow meter is not installed, it is estimated by a time series neural network with raw water flow. Figure 5 shows the system configuration in which the neuro-fuzzy controller provides the chlorine injection rate considering target output residual chlorine, filtered residual chlorine and input residual chlorine. Then, the predicted dosage rate is multiplied with the estimated outflow to compute the injection quantity. The current input residual chlorine is observed by an internal secondary feedback loop to modify the bias value of neuro-fuzzy algorithm. The output residual chlorine is monitored continuously only to change the additional bias in the first feedback loop.

Figure 6 shows a software program of a hybrid controller that was and is being applied to a water treatment plant. The program was connected to SCADA (Supervisory Control and Data Acquisition) System with OPC (OLE for Process Control) protocol to obtain the desired data. If a tag name is entered into the software, the values are acquired in any SCADAs to support OPC protocol. In addition, there are functions to prevent

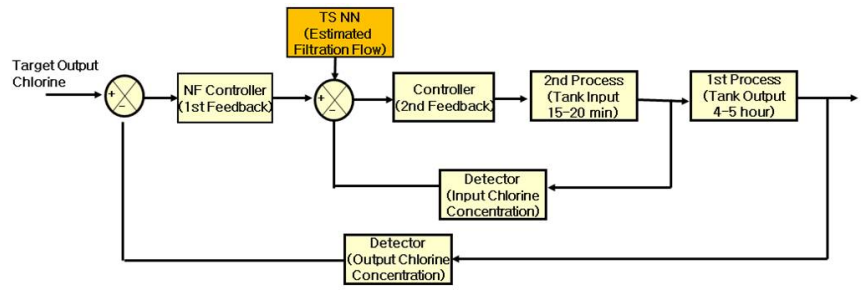

Figure 5. Diagram of a hybrid TS-NF controller.

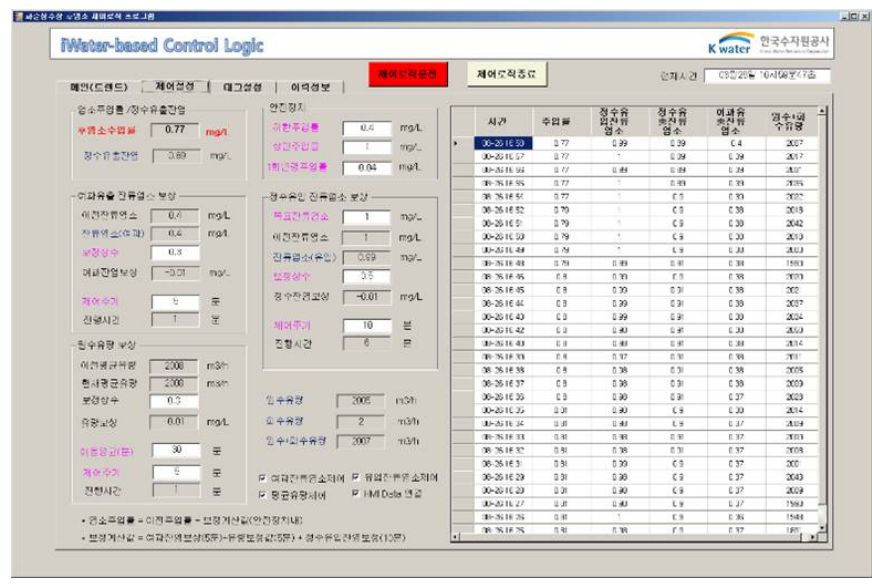

Figure 6. Implementation of a post-chlorination controller.

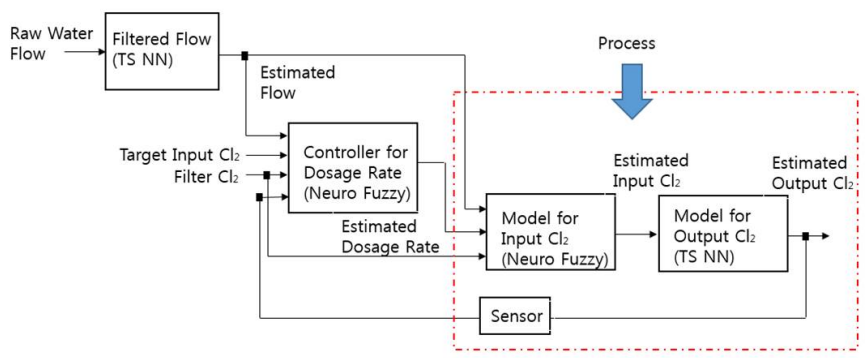

Figure 7. Simulation process.

incorrect injection rate accidents from occurring, such as low and high limits and one time change rates, which are set based on an operator's experience.

\section{Simulation and Experimental Results}

The algorithm procedures are shown in Figure 7 for the control logic simulation. At first, the time series neural network was applied to predict the filtration flow through the raw water flow. Then, the controller is designed to determine the optimal injection rate through the neural net algorithm, which also leads to the estimation of input and output residual chlorine. 


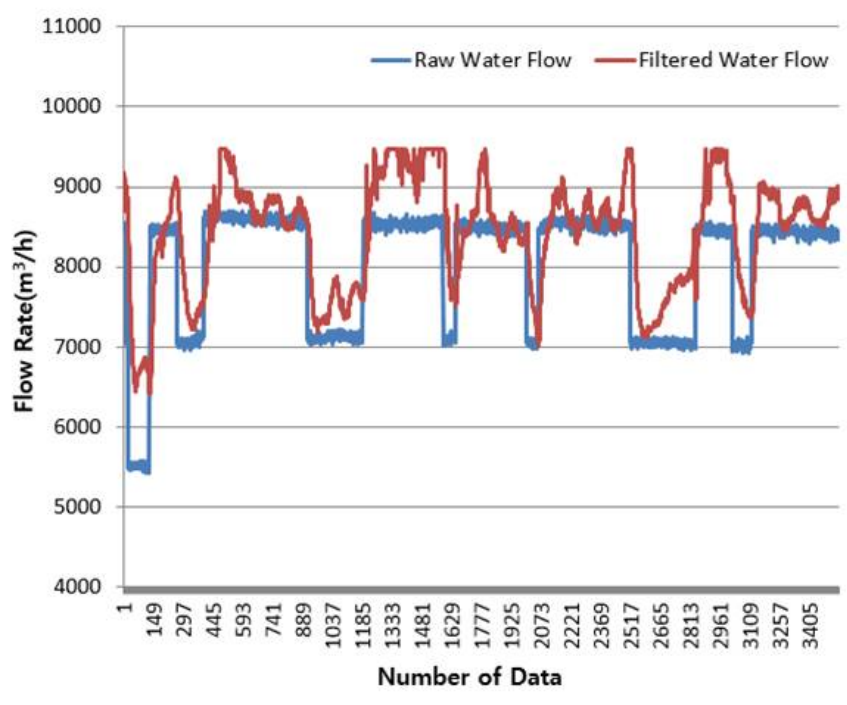

Figure 8. Comparison of raw and filtered flow.

\subsection{Prediction of Filtration Flow}

Typical time-series analysis and pattern recognition algorithms are reviewed and compared to estimate the filtered flow based on the raw water flow. In case of no filtration flow in a water treatment plant, the flow is generally distorted by using raw water flow, whose phenomenon is shown in Figure 8. The figures are compared with the true raw and filtered water, which are installed in a plant. It shows that the difference between two meters happens in many places.

\subsubsection{Moving average}

In Figure 9, filtered flow is estimated by the simple moving average, in which the flow has the same trend as the raw water flow in the region of rapid change. However, the follow-up after sharply rising and falling flow did not go smoothly.

Figure 10 shows the result to compute the optimal time in the moving average, of which the lowest percentage error (4.88\%) occurs at 41 minutes.

\subsubsection{Time series neural network}

Raw water flow data is used for 1-10 minutes ago as linear regression in the algorithm to estimate the flow. Figure 11 shows that it is estimated better both in the regions of sudden and small change compared to the other algorithms.

As the algorithm increases the delay time by one minute slowly, the percentage error improves in contrast to the moving average of 41 minutes shown in Figure 12, in which 87 minutes

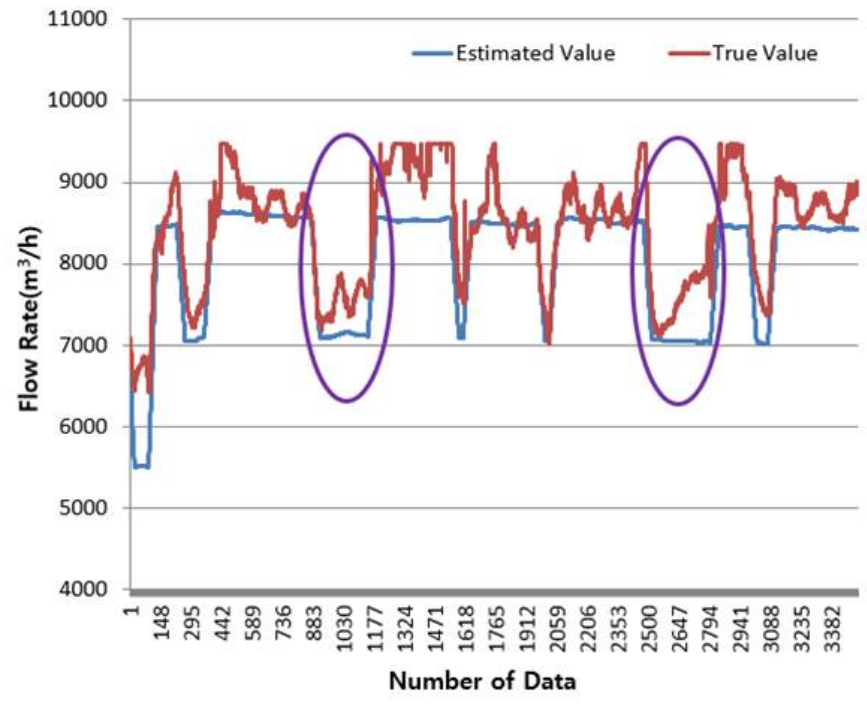

Figure 9. Flow estimation based on simple moving average.

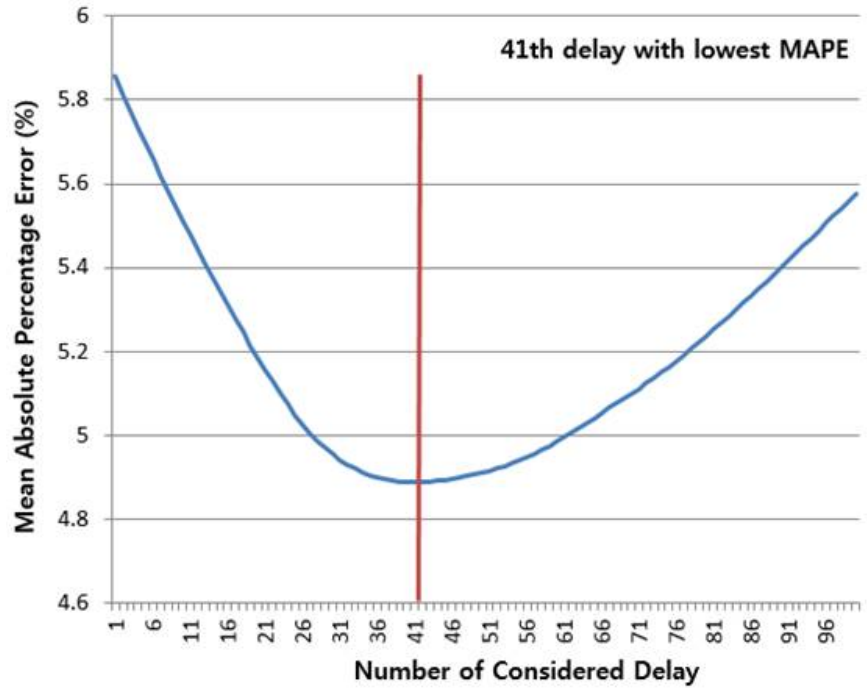

Figure 10. MAPE of simple moving average.

delay has the lowest error at $2.72 \%$. However, the improvement is not significant after 40 minute delay.

\subsection{Controller for Determining Chlorine Dosage Rate}

Residual chlorine concentrations in filtration and input storage tank and estimated filtered flow are considered for determining the chlorine injection rate. The rate has been reviewed by linear and nonlinear learning algorithms. The results are shown in Tables 1 , in which the neuro-fuzzy algorithm is 2.38 times better than the linear regression algorithm.

Figure 13 is estimated by neuro fuzzy algorithm, which 


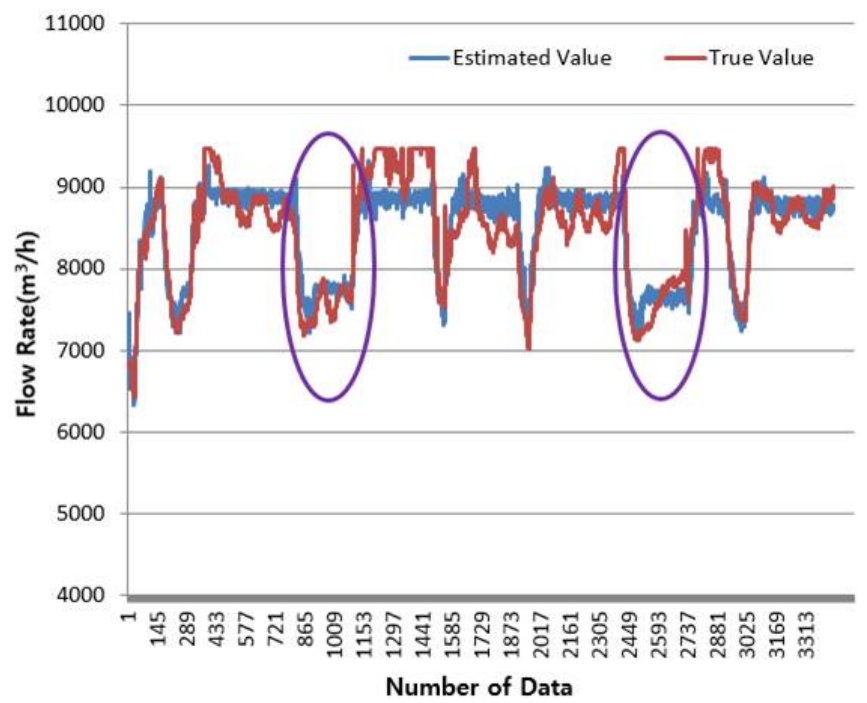

Figure 11. Flow estimation by time series neural network.

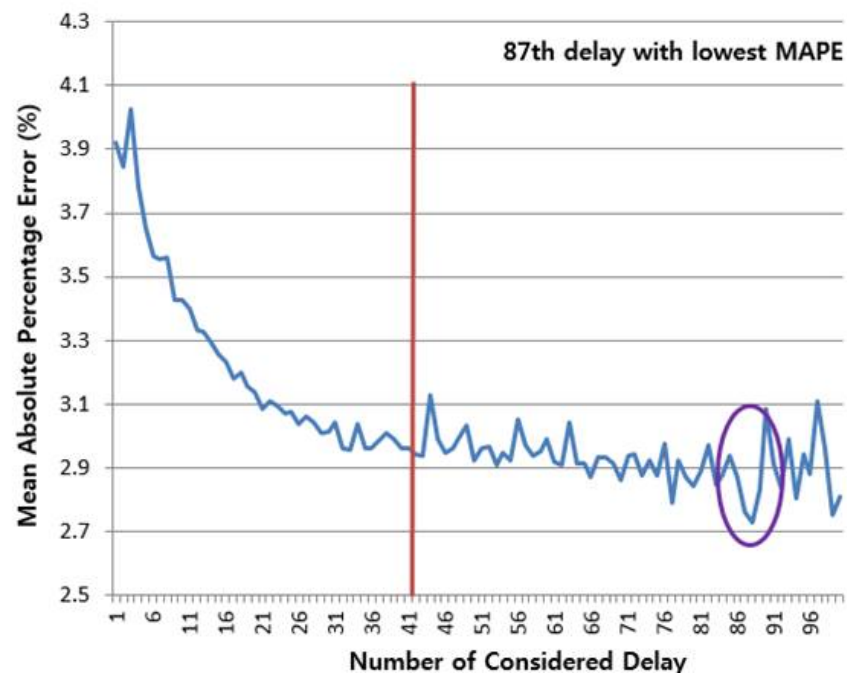

Figure 12. MAPE of time series neural network.

Table 1. Algorithm comparison for the controller

\begin{tabular}{ccc}
\hline Algorithm & $\begin{array}{c}\text { Multi linear } \\
\text { regression }\end{array}$ & Neuro-fuzzy \\
\hline MAPE & 4.372 & 1.831 \\
MAE & 0.021 & 0.009 \\
RMSE & 0.028 & 0.013 \\
\hline
\end{tabular}

shows that it renovates the unexplainable error in the linear regression. Thus, the nonlinear algorithm is selected for the implementation of post-chlorination.

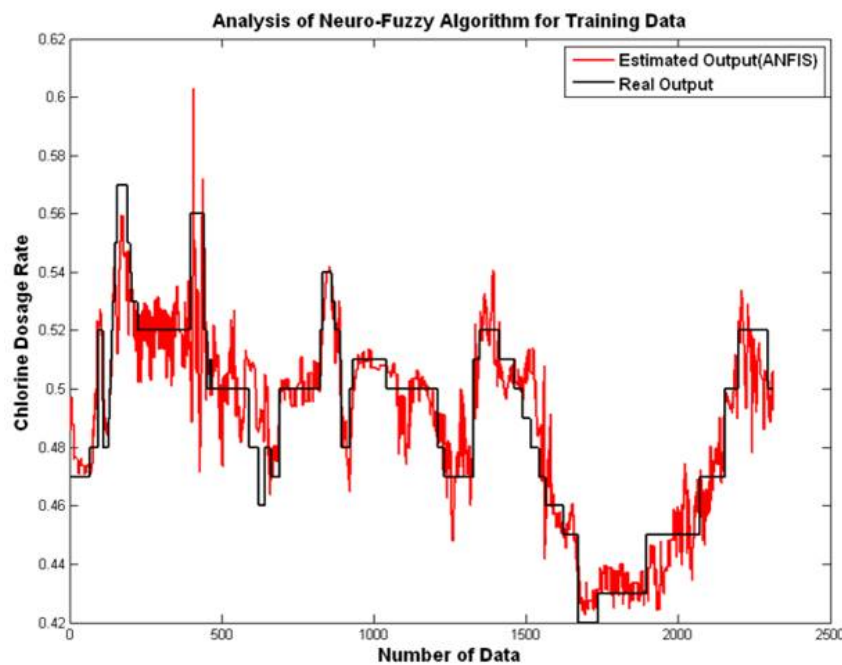

Figure 13. Estimation results by neuro-fuzzy algorithm.

Table 2. Algorithm comparison for residual chlorine modeling

\begin{tabular}{ccc}
\hline Algorithm & $\begin{array}{c}\text { Multi linear } \\
\text { regresssion }\end{array}$ & Neuro-fuzzy \\
\hline MAPE & 2.099 & 1.284 \\
MAE & 0.021 & 0.013 \\
RMSE & 0.028 & 0.018 \\
\hline
\end{tabular}

\subsection{Modeling for Input Residual $\mathrm{Cl}_{2}$}

Modeling is implemented to be verified before the controller is applied to water treatment process. It will estimate the input residual chlorine in case the dosage rate changes, residual chlorine concentrations and estimated filtered flow. Table 2 shows that the neuro fuzzy algorithm is 1.63 times better in comparison to the linear algorithm.

\subsection{Modeling for Output Residual $\mathrm{Cl}_{2}$}

The ultimate goal is to keep the output residual chlorine rate constant in post-chlorination. However, it is not sure due to the inconstant residence time, even though the current value of the chlorine injection rate and input residual chlorine are fair. Because the relationship is not clear between the input and output chlorine rates, the relationship should be analyzed by data mining techniques. Table 3 shows the results of the correlation analysis between the input and output, in which the coefficient is as low as 0.12 in 0 minute, no moving average, but the result in $-120 \sim-60$ minute with a moving average of 0.92 which is significantly good.

Figure 14 compares the input and output chlorine rates, where 
Table 3. Correlation analysis of residual chlorine considering delay time

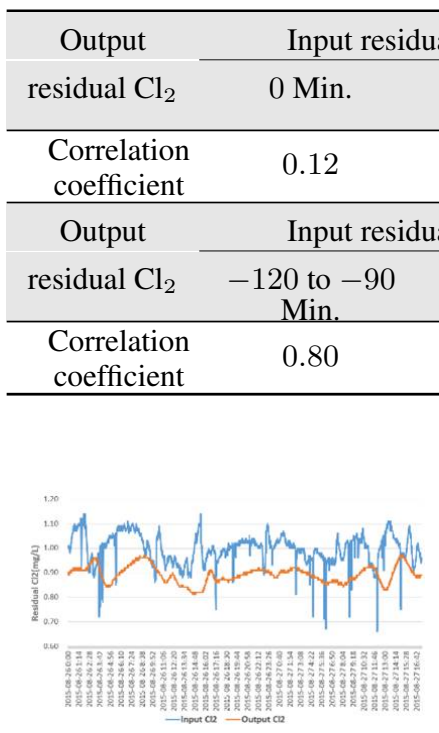

(a)

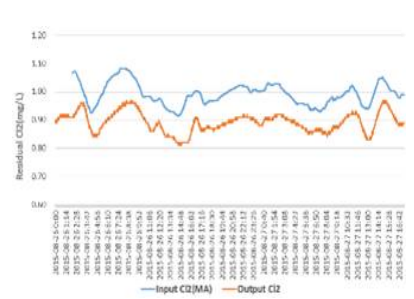

(b)
Figure 14. Comparison of input and output chlorine rate (a) input and output chlorine rate without moving average; (b) input and output chlorine rate with moving average.

Table 4. Error comparison in consideration of bias

\begin{tabular}{ccc}
\hline & Without bias & With bias \\
\hline MAPE & 3.4055 & 3.2871 \\
MAE & 0.0341 & 0.0329 \\
RMSE & 0.0126 & 0.0394 \\
\hline
\end{tabular}

Figure 14(a) does not consider any delay time, but Figure 14(b) considers a 90 minutes delay in the output chlorine rate, where the input data are averaged from -120 to -60 minutes. The total averages of the input and output are $0.99 \mathrm{mg} / \mathrm{L}$ and $0.89 \mathrm{mg} / \mathrm{L}$, which means the evaporation is $0.1 \mathrm{mg} / \mathrm{L}$ in the tank. Figure 14(b) is easily explainable in terms of evaporation compared to Figure 14(a), which has the abnormal data with a sudden fall in the input chlorine.

\subsection{Simulation Results}

The simulation is performed on the basis of the proposed modeling. The error of the input chlorine rate is reviewed in Table 4, in which MAPE in case of "Without bias" is 3.4\%. If the error of the output chlorine rate is fed back to target input chlorine rate like "With bias", the error decreases to $3.28 \%$.

The simulation results in Figure 15 show that the target resid-

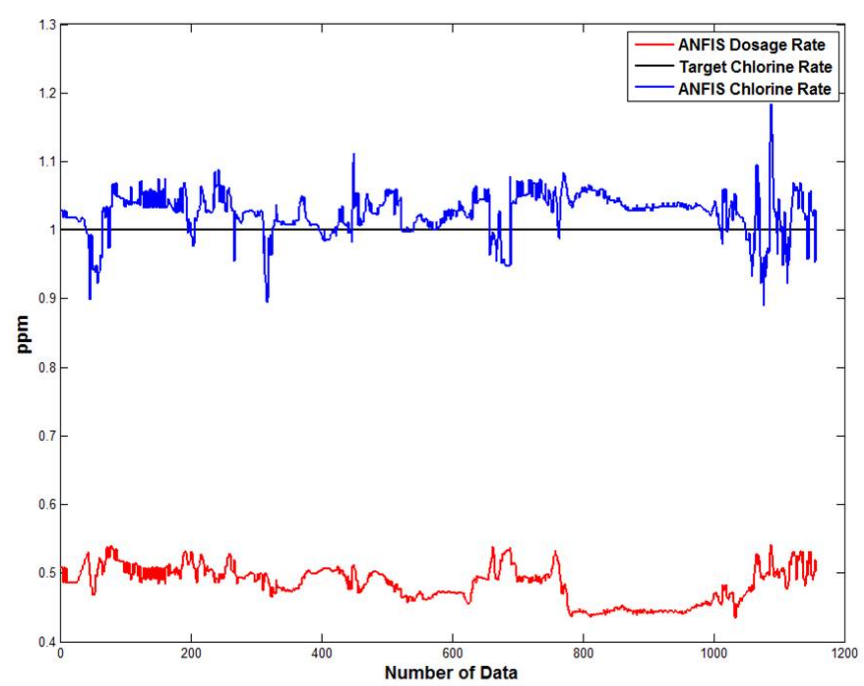

Figure 15. Simulation results without feedback.

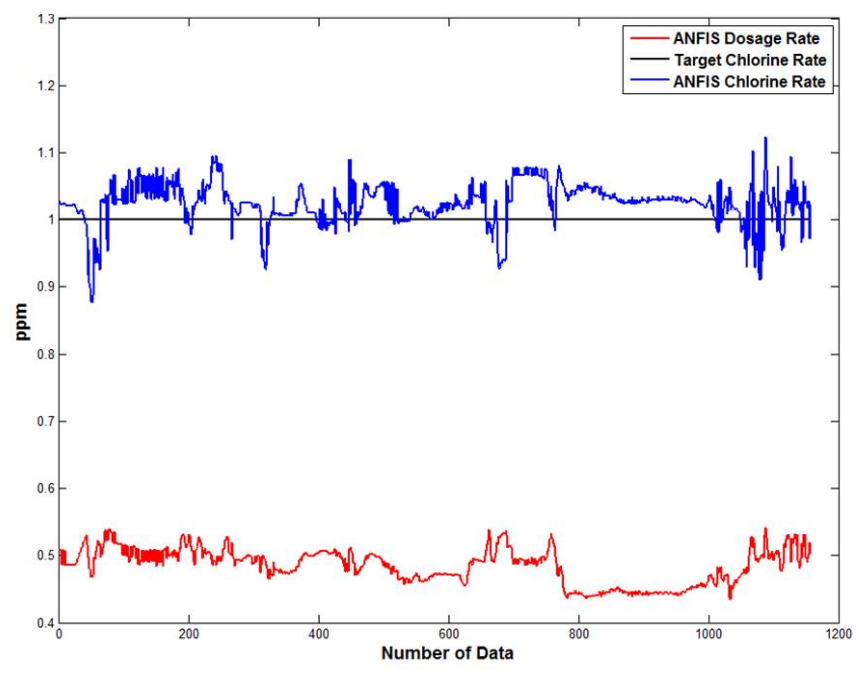

Figure 16. Simulation results with feedback.

ual chlorine rate is reached by the controller learned by neurofuzzy algorithm. The chlorine rate estimated by the algorithm appears slightly higher than the desired residual chlorine. It is considered due to the additional disturbance to be excluded. In particular, the region of 1,000 to 1,500 th data set has a maximum error of $0.2 \mathrm{ppm}$.

The results in Figure 16 show that the method of cascade control is applied to improve the large error in Figure 20, by which the region of 1,000 to 1,500th data the error decreases significantly from $0.2 \mathrm{ppm}$ to $0.1 \mathrm{ppm}$.

\subsection{Experimental Results in the WTP}

The developed control logic program is applied to the operating water treatment plant to check the practical effectiveness. "A" 
Table 5. Effects of the proposed algorithm

\begin{tabular}{lcccc}
\hline \multirow{2}{*}{ Process } & \multicolumn{2}{c}{ Old algorithm } & \multicolumn{2}{c}{ Proposed algorithm } \\
\cline { 2 - 5 } & Mid. & Post & Mid. & Post \\
\hline $\begin{array}{l}\text { Standard } \\
\text { deviation }\end{array}$ & 0.061 & 0.031 & 0.035 & 0.019 \\
MAPE & $11.50 \%$ & $2.80 \%$ & $5.84 \%$ & $1.60 \%$ \\
\hline
\end{tabular}

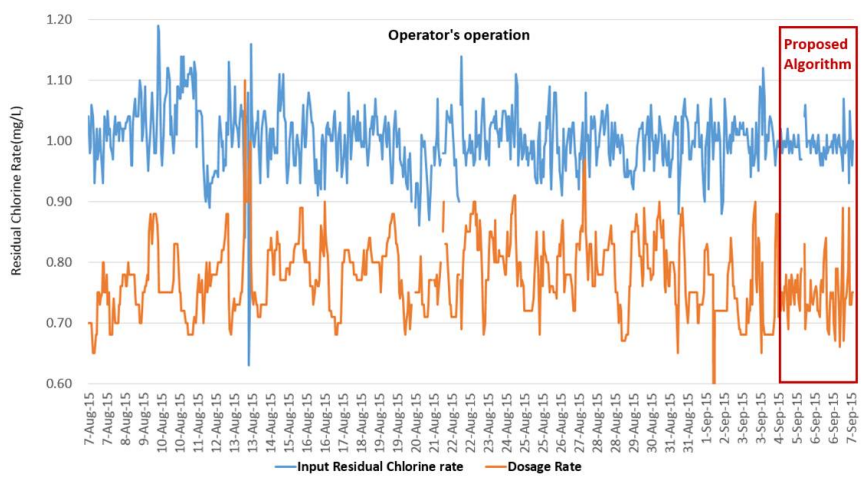

Figure 17. Improvement of the input residual chlorine variation.

water purification plant in South Jeolla province is chosen as the testbed site, because the plant has chlorine control difficulties and no filtration flow. In order to assess the effect of improving the water quality, the proposed algorithm is evaluated whether the target input and output residual chlorine rates are reached. Table 5 shows the effects of the proposed algorithm, by which the standard deviation and percentage error improved by 1.75 times. In addition, the deviation and error are 1.96 times better in mid chlorination, in which filtration flow estimation and feedback control are used.

Figure 17 shows the improvement results of the input chlorine concentration, in which the input chlorine rate (blue line) improves significantly in variance within the red box, which is operated by the proposed algorithm during the period from Sep. 4 to Sep. 7, 2015. At that time, the dosage rate (orange line) changed very frequently and automatically by the algorithm to keep the target value.

Controller for the dosage rate and modeling for the output residual chlorine are estimated by neuro fuzzy algorithm, which is used to predict the non-linear function. As shown in Figure 18, the application of the proposed algorithm significantly improves the achievement of the target residual chlorine rate by $0.9 \mathrm{ppm}$. The variance becomes smaller by the proposed controller.

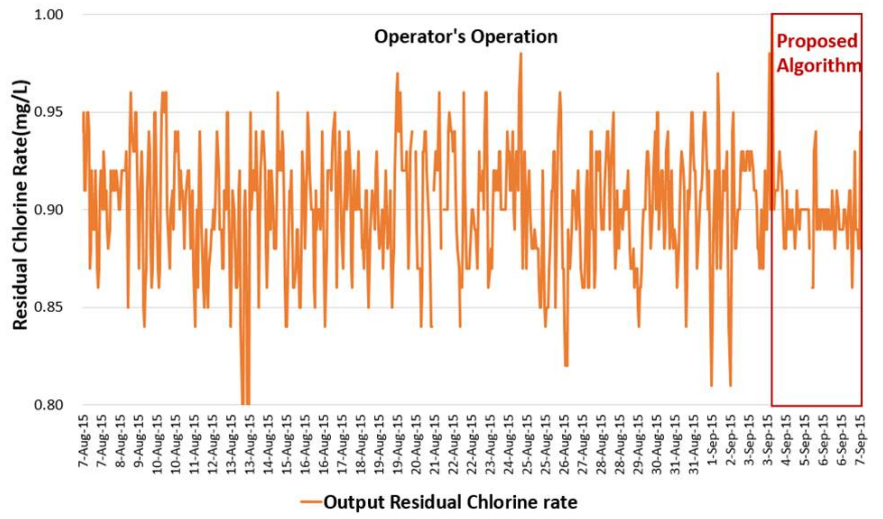

Figure 18. Improvement of the output residual chlorine variation.

\section{Conclusion}

In case that a raw water flow meter is used for post-chlorination in a plant without a filtered flow meter owing to improper installation situations, many of the difficulties have currently been caused in the chemical dosage. A hybrid neuro fuzzy algorithm is proposed and applied to the operating water treatment process, in which the filtration flow rate is estimated by a time series neural network and the neuro-fuzzy algorithm is applied as the controller for the optimal chemical dosage rate and the modeling for the residual chlorine rate estimation. The proposed algorithm leads to water quality improvements in post chlorination and mid chlorination of 1.75 times and 1.96 times respectively. It can also reduce 0.3 billion dollars economically to install flow meters, pipeline correction and manholes. It also enables hygienically safe drinking water supply with preemptive response for the time delay, inherent characteristics of the disinfection process

\section{References}

[1] Korean Water and Wastewater Works Association, "Water treatment process," in Facility Standard for Water Treatment Process. Gwacheon: Ministry of Environment, 2010, pp. $429-430$.

[2] Korean Water and Wastewater Works Association, 463023070"Standards for water supply facilities," in $\mathrm{Fa}$ cility Standard for Water Treatment Process. Gwacheon: Ministry of Environment, 2010, pp. 449-451.

[3] J. Kim and J. A. Han, "Rechlorination for residual chlorine concentration equalization in distribution system," Journal of Korean Society of Water and Wastewater, vol. 28, no. 1, 
pp. 91-101, 2014. http://dx.doi.org/10.11001/jksww.2014. 28.1.91

[4] D. L. Boccelli, M. E. Tryby, J. G. Uber, and R. S. Summers, "A reactive species model for chlorine decay and THM formation under rechlorination conditions," $\mathrm{Wa}$ ter Research, vol. 37, no. 11, pp. 2654-2666, 2003. http://dx.doi.org/10.1016/S0043-1354(03)00067-8

[5] G. W. Shin and K. H. Lee, "The development of prediction model to control post chlorination process," Journal of Korean Society of Water and Wastewater, vol. 22, no. 5, pp. 213-218, 2008.

[6] K. H. Lee, J. H. Kim, J. L. Lim, and S. H. Chae, "The development of prediction model to keep the sedimentation chlorine rate constant in pre chlorination," Journal of Korean Society of Water and Wastewater, vol. 21, no. 5, pp. 601-607, 2007.

[7] J. C. Ahn, S. W. Lee, B. S. Rho, Y. J. Choi, J. H. Choi, H. I. Kim, et al., "Modeling residual chlorine and THMs in water distribution system," Journal of Korean Society of Environmental Engineers, vol. 29, no. 6, pp. 706-714, 2007.

[8] D. R. Broad, G. C. Dandy, and H. R. Maier, "Water distribution system optimization using metamodels," Journal of Water Resources Planning and Management, vol. 131, no. 3, pp. 172-180, 2005. http://dx.doi.org/10.1061/(ASCE) 0733-9496(2005)131:3(172)

[9] V. K. Chambers, J. D. Creasey, and J. S. Joy, "Modeling free and total chlorine decay in potable water distribution-systems," Journal of Water Supply Research and Technology-Aqua, vol. 44, no. 2, pp. 60-69, 1995.

[10] F. Hua, J. R. West, R. A. Barker, and C. F. Forster, "Modelling of chlorine decay in municipal water supplies," Water Research, vol. 33, no. 12, pp. 2735-2746, 1999. http://dx.doi.org/10.1016/S0043-1354(98)00519-3

[11] Korean Water and Wastewater Works Association, "Water treatment process," in Facility Standard for Water Treatment Process. Gwacheon: Ministry of Environment, 2010, p. 467.

[12] S. T. Hong, A. K. Lee, H. H. Lee, N. S. Park, and S. W. Lee, "Application of neuro-fuzzy PID controller for effective post-chlorination in water treatment plant," Desalina- tion and Water Treatment, vol. 47, no. 1-3, pp. 211-220, 2012. http://dx.doi.org/10.1080/19443994.2012.696810

[13] F. Rosenblatt, Principles of Neurodynamics: Perceptrons and the Theory of Brain Mechanisms. Washington, DC: Spartan, 1962.

[14] E. Alpaydin, Introduction to Machine Learning. Cambridge, MA: MIT Press, 2004.

[15] D. E. Rumelhart, G. E. Hinton, and R. J. Williams, Learning Internal Representations by Error Propagation, La Jolla, CA: Institute for Cognitive Science, University of California-San Diego, 1985.

[16] H. H. Lee, S. B. Jang, G. W. Shin, S. T. Hong, D. J. Lee, and M. G. Chun, "An ultrasonic multi-beam concentration meter with a neuro-fuzzy algorithm for water treatment plants," Sensors, vol. 15, no. 10, pp. 26961-26977, 2015. http://dx.doi.org/10.3390/s151026961

[17] J. S. R. Jang, "ANFIS: adaptive-network-based fuzzy inference system," IEEE Transactions on Systems, Man, and Cybernetics, vol. 23, no. 3, pp. 665-685, 1993. http://dx.doi.org/10.1109/21.256541

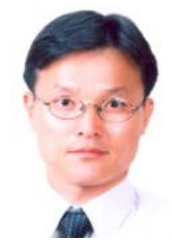

HoHyun Lee received his B.S. from Department of Electronics Engineering, Wonkwang University and M.S. from Department of Robotics Engineering, KAIST, Korea, in 1998 and 2010, respectively. He worked at K-water from 1998 to present. His research interests include fuzzy, pattern recognition and optimization.

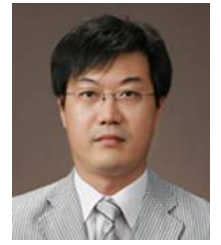

GangWook Shin received his B.S. from Department of Electronics Engineering, Dongguk University and M.S. and Ph.D. from Department of Electronics Engineering, Hongik University, Korea, in 1987, 1993 and 2005, respectively. He worked at K-water from 1993 to present. His research interests include modeling, machine learning and process control.

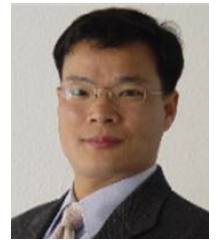

SungTaek Hong received his B.S. and M.D. from Department of Electronics Engineering, and Hanbat University, Korea, in 1993 and 1995, respectively. He worked at K-water from 1996 to present. His research interests include satellite, intelligent control and cognitive science. 


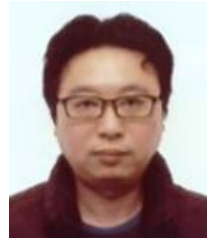

JongWoong Choi received his M.S. and Ph.D. from Department of Mechanical Engineering, Korea Maritime and Ocean University in 2001 and 2012, respectively. He worked at $\mathrm{K}$-water from 2015 to present. His research interests include hydraulic machine and water treatment plant.

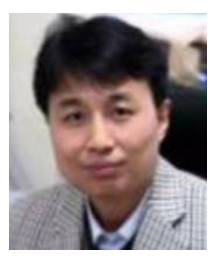

MyungGeun Chun is a professor in Chungbuk National University. He received his B.S. from Department of Electronics Engineering, Busan University and M.S., Ph.D. from Department of Electric and Electronic Engineer- ing, KAIST, Korea, in 1987, 1989 and 2003, respectively. He worked as a researcher in Samsung Electronics. He has been review board in ISO/IEC SC 27 since 2007 and TTA PG 505 since 2008. His research interests include datamining, information security and image processing. 